Journal of Computer Science 4 (9): 741-743, 2008

ISSN 1549-3636

(C) 2008 Science Publications

\title{
The Relationship between Exclusive-Or and the Unique Existential Quantifier
}

\author{
Maher A. Nabulsi and Ayman M. Abdalla \\ Department of Computer Science, Al-Zaytoonah University of Jordan, \\ Amman 11733, Jordan
}

\begin{abstract}
Problem Statement: The efficiency of computer architecture design is affected by the components used. Establishing a relationship between exclusive-or (XOR) and the unique existential quantifier provides alternative system implementations. Approach: Applications of XOR and the unique existential quantifier were explained. Then, prepositional logic was used to establish the relationship between them. Results: Different quantified assertions with two variables that use the unique existential quantifier were represented without quantifiers by using XOR. Conclusions: The unique existential quantifier and XOR were helpful in some computer architecture systems such as multiplexers, decoders and bus systems. The unique existential quantifier and XOR may be used interchangeably in some situations, but not always.
\end{abstract}

Key words: Exclusive-or, multiplexer, decoder, unique existential quantifier, universal quantifier

\section{INTRODUCTION}

This study establishes a relationship between exclusive-or (XOR) and the unique existential quantifier ( $\exists$ !). The representations of the unique existential quantifier in computer architecture may be done using any of several combinations of XOR gates, multiplexers, decoders, AND gates and OR gates. This is closely related to propositional logic, where computer architecture circuits may be represented with logical formulas.

\section{MATERIALS AND METHODS}

Several applications in computer architecture implicitly apply $\exists$ !xP(x), mainly in situations that use a bus system and need to take information from one source while all other sources are inhibited. For example, consider the predicate $\mathrm{P}(\mathrm{x})$ defined over the finite set $\{1,2,3,4\}$. Then, $\exists ! \mathrm{xP}(\mathrm{x})$ is logically equivalent to:

$$
\begin{aligned}
& {[\mathrm{P}(1) \wedge \sim \mathrm{P}(2) \wedge \sim \mathrm{P}(3) \wedge \sim \mathrm{P}(4)] \vee} \\
& {[\sim \mathrm{P}(1) \wedge \mathrm{P}(2) \wedge \sim \mathrm{P}(3) \wedge \sim \mathrm{P}(4)] \vee} \\
& {[\sim \mathrm{P}(1) \wedge \sim \mathrm{P}(2) \wedge \mathrm{P}(3) \wedge \sim \mathrm{P}(4)] \vee} \\
& {[\sim \mathrm{P}(1) \wedge \sim \mathrm{P}(2) \wedge \sim \mathrm{P}(3) \wedge \mathrm{P}(4)] .}
\end{aligned}
$$

This means that only one of the four possible elements of $\mathrm{P}(\mathrm{x})$ can be true, while the others must all be false. This idea can be seen in various applications.
A $4 \times 1$ multiplexer (MUX), also known as a data selector, is illustrated in Fig. 1. The value of the output; $\mathrm{U}$, is equal to one of the inputs; $\mathrm{I}_{0}$ through $\mathrm{I}_{4}$, selected based on the controls; $\mathrm{S}_{0}$ and $\mathrm{S}_{1}$. These values are shown in Table 1.

A decoder with three-state buffers, seen in Fig. 2, selects one of the data inputs; $\mathrm{I}_{0}$ through $\mathrm{I}_{7}$, corresponding to the control inputs, $\mathrm{D}_{0}$ through $\mathrm{D}_{7}$. These control inputs are determined by a decoder controlled by three variables, as shown in Table 2. Only one data input is transferred to the single output at any given time.

Decoders and multiplexers are important building blocks of computer systems ${ }^{[4,5]}$. For example, a bus system may be constructed using a system of decoders with three-state buffers or using multiplexers. The single output of each multiplexer (or decoder system) represents one line in the bus. This bus has various applications, such as the system seen in Fig. 3. In this example, the system uses four multiplexers to choose a 4-bit word from one of four memories (A, B, C and D). The selection lines, $\mathrm{S}_{0}$ and $\mathrm{S}_{1}$, control the multiplexers so that one memory word is selected, as shown in Table 3 .

XOR has applications in logic programming ${ }^{[1,2]}$ and in experimental psychology ${ }^{[3]}$. In addition, XOR may be used to implement a one-selecting variant of a transition system ${ }^{[2]}$, which may also be represented using the unique existential quantifier.

Corresponding Author: Maher A. Nabulsi, Department of Computer Science, Al-Zaytoonah University of Jordan, Amman 11733, Jordan 


\begin{tabular}{lll} 
Table 1: Output of multiplexer selected from the inputs \\
\hline$S_{1}$ & $\mathrm{~S}_{0}$ & $\mathrm{U}$ \\
\hline 0 & 0 & $\mathrm{I}_{0}$ \\
0 & 1 & $\mathrm{I}_{1}$ \\
1 & 0 & $\mathrm{I}_{2}$ \\
1 & 1 & $\mathrm{I}_{3}$ \\
\hline
\end{tabular}

Table 2: Output of buffer system selected from the inputs

\begin{tabular}{lllcl}
\hline $\mathrm{S}_{2}$ & $\mathrm{~S}_{1}$ & $\mathrm{~S}_{0}$ & Active output of decoder & $\mathrm{U}$ \\
\hline 0 & 0 & 0 & $\mathrm{D}_{0}$ & $\mathrm{I}_{0}$ \\
0 & 0 & 1 & $\mathrm{D}_{1}$ & $\mathrm{I}_{1}$ \\
0 & 1 & 0 & $\mathrm{D}_{2}$ & $\mathrm{I}_{2}$ \\
0 & 1 & 1 & $\mathrm{D}_{3}$ & $\mathrm{I}_{3}$ \\
1 & 0 & 0 & $\mathrm{D}_{4}$ & $\mathrm{I}_{4}$ \\
1 & 0 & 1 & $\mathrm{D}_{5}$ & $\mathrm{I}_{5}$ \\
1 & 1 & 0 & $\mathrm{D}_{6}$ & $\mathrm{I}_{6}$ \\
1 & 1 & 1 & $\mathrm{D}_{7}$ & $\mathrm{I}_{7}$ \\
\hline
\end{tabular}

Table 3: Memory word selected for bus lines based on the controls

\begin{tabular}{lll}
\hline $\mathrm{S}_{1}$ & $\mathrm{~S}_{0}$ & Selected memory word \\
\hline 0 & 0 & From Memory A \\
0 & 1 & From Memory B \\
1 & 0 & From Memory C \\
1 & 1 & From Memory D \\
\hline
\end{tabular}

Table 4: Truth table for the unique existential quantifier and XOR with two elements

\begin{tabular}{llll}
\hline $\mathrm{P}(1)$ & $\mathrm{P}(2)$ & $\exists ! \mathrm{xP}(\mathrm{x})$ & $\mathrm{P}(1) \oplus \mathrm{P}(2)$ \\
\hline 0 & 0 & 0 & 0 \\
0 & 1 & 1 & 1 \\
1 & 0 & 1 & 1 \\
1 & 1 & 0 & 0 \\
\hline
\end{tabular}

Table 5: Truth table for the unique existential quantifier and XOR with three elements

\begin{tabular}{lllll}
\hline $\mathrm{P}(1)$ & $\mathrm{P}(2)$ & $\mathrm{P}(3)$ & $\exists ! \mathrm{xP}(\mathrm{x})$ & $\mathrm{P}(1) \oplus \mathrm{P}(2) \oplus \mathrm{P}(3)$ \\
\hline 0 & 0 & 0 & 0 & 0 \\
0 & 0 & 1 & 1 & 1 \\
0 & 1 & 0 & 1 & 1 \\
0 & 1 & 1 & 0 & 0 \\
1 & 0 & 0 & 1 & 1 \\
1 & 0 & 1 & 0 & 0 \\
1 & 1 & 0 & 0 & 0 \\
1 & 1 & 1 & 0 & 1 \\
\hline
\end{tabular}

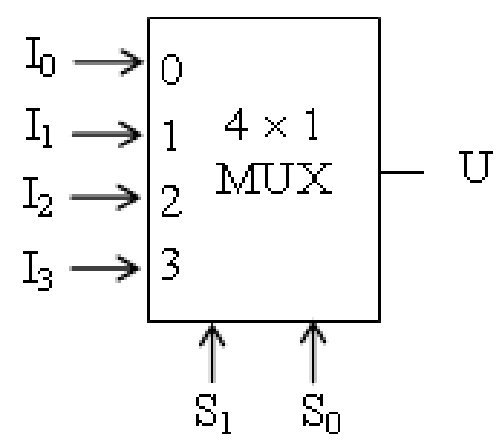

Fig. 1: A $4 \times 1$ Multiplexer

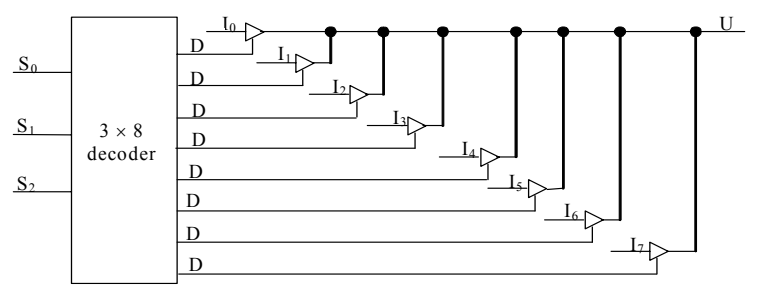

Fig. 2: A decoder with three-state buffers, where the outputs of the decoder (from the top down) are $\mathrm{D}_{0}$ through $\mathrm{D}_{7}$

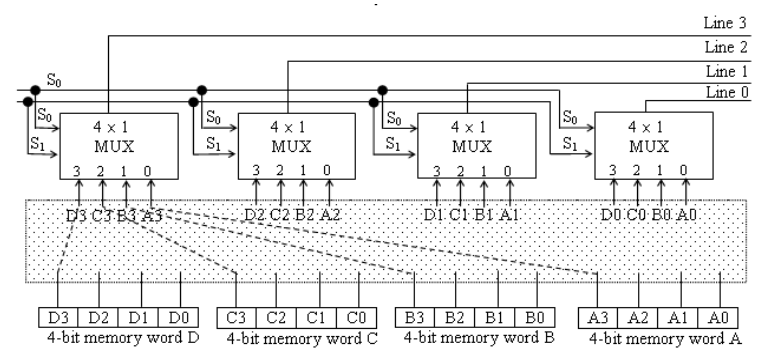

Fig. 3: A memory selection system using multiplexers

The relationship between XOR and the unique existential quantifier can be seen by examining truth tables. First, consider a predicate $\mathrm{P}$ defined over a finite universe of one variable (x) with two elements $\{1,2\}$. As seen in Table 4, $\exists$ ! $\mathrm{xP}(\mathrm{x})$ is equivalent to $\mathrm{P}(1) \oplus$ $\mathrm{P}(2)$. However, with a finite universe of three elements $\{1,2,3\}, \exists$ ! $\mathrm{xP}(\mathrm{x})$ is not equivalent to $\mathrm{P}(1) \oplus \mathrm{P}(2) \oplus$ $\mathrm{P}(3)$. As seen in Table 5, they do not have the same value when all three elements are true. Furthermore, $\exists ! \mathrm{XP}(\mathrm{x})$ is not equivalent to XOR with a number of elements of four or more. This is because XOR is an odd function that has a value of 1 when there is an odd number of elements (possibly three or more) with the value 1 . The unique existential quantifier, on the other hand, has a value of 1 only when there is exactly one element with the value 1 .

With a function of two variables; $\mathrm{x}$ and $\mathrm{y}$, and a universe of two elements; $\{1,2\}$, quantified assertions can be represented using XOR. For example, consider the following two assertions.

$$
\begin{aligned}
\forall \mathrm{x} \exists ! \mathrm{yP}(\mathrm{x}, \mathrm{y}) \Leftrightarrow & ([\mathrm{P}(1,1) \wedge \sim \mathrm{P}(1,2)] \vee[\mathrm{P}(1,2) \\
& \wedge \sim \mathrm{P}(1,1)]) \wedge([\mathrm{P}(2,1) \wedge \sim \mathrm{P}(2,2)] \\
& \vee[\mathrm{P}(2,2) \wedge \sim \mathrm{P}(2,1)]), \\
\exists ! \mathrm{y} \forall \mathrm{xP}(\mathrm{x}, \mathrm{y}) \Leftrightarrow \quad & ([\mathrm{P}(1,1) \wedge \mathrm{P}(2,1)] \wedge \sim[\mathrm{P}(1,2) \\
& \wedge \mathrm{P}(2,2)]) \vee([\mathrm{P}(1,2) \wedge \mathrm{P}(2,2)] \\
& \wedge \sim[\mathrm{P}(1,1) \wedge \mathrm{P}(2,1)]) .
\end{aligned}
$$


Since $\mathrm{A} \oplus \mathrm{B}$ is equivalent to $(\sim \mathrm{A} \wedge \mathrm{B}) \vee(\mathrm{A} \wedge \sim \mathrm{B})$, the above assertions can be written as:

$$
\begin{aligned}
& \forall \mathrm{x} \exists ! \mathrm{yP}(\mathrm{x}, \mathrm{y}) \Leftrightarrow[\mathrm{P}(1,1) \oplus \mathrm{P}(1,2)] \wedge[\mathrm{P}(2,1) \oplus \mathrm{P}(2,2)], \\
& \exists ! \mathrm{y} \forall \mathrm{xP}(\mathrm{x}, \mathrm{y}) \Leftrightarrow[\mathrm{P}(1,1) \wedge \mathrm{P}(2,1)] \oplus[\mathrm{P}(1,2) \wedge \mathrm{P}(2,2)] .
\end{aligned}
$$

\section{RESULTS}

The values of $\exists$ ! $\mathrm{xP}(\mathrm{x})$ and $\mathrm{XOR}$ are equivalent for two elements, but not for three or more elements.

The quantified assertions $\forall \mathrm{x} \exists$ !yP $(\mathrm{x}, \mathrm{y})$ and $\exists ! y \forall \mathrm{xP}(\mathrm{x}, \mathrm{y})$ on the universe of two elements can be written through XOR and AND functions because the universal quantifier $(\forall)$ is equivalent to $\mathrm{AND}$, and the unique existential quantifier is equivalent to XOR.

\section{DISCUSSION}

XOR and the unique existential quantifier are interchangeable for two elements. This helps redesign some architecture systems to increase their efficiency. In addition, some logical equations that use quantified assertions can be simplified using AND and XOR.

The idea of the unique existential quantifier is clearly seen in a decoder where only one output is active at any given time, while all other outputs are inactive. The decoder is a part of the multiplexer, and therefore, the idea of the unique existential quantifier can be used in different applications that use decoders and multiplexers. This includes multiple processor and multiple memories connected to bus systems.

\section{CONCLUSIONS}

The relationship between $\exists$ !xP(x) and XOR was discussed, and some applications of $\exists ! \mathrm{xP}(\mathrm{x})$ in computer architecture were presented.

There are several hardware applications for $\exists ! \mathrm{PP}(\mathrm{x})$ in computer architecture, such as in a multiplexer, in a decoder with three state buffers and in a data bus with multiple memories.

\section{REFERENCES}

1. Cho, S.J., U.S. Choi, Y.H. Hwang and H.D. Kim, 2008. Design of new XOR-based hash functions for chache memories. Computers and Math. Appl., 55: 2005-2011. DOI: 10.1016/j.camwa.2007.07.008

2. Fecher, H. and H. Schmidt, 2008. Comparing disjunctive modal transition systems with an oneselecting variant. J. Logic and Algebraic Programming, 77: 20-39. DOI: 10.1016/j.jlap.2008.05.003

3. Grand, C. and R.C. Honey, 2008. Solving XOR. J. Exp. Psy. Anim. Behavior Process., 34: 486-493. http://www.ncbi.nlm.nih.gov/pubmed/18954232

4. Khan, M., 2007. Reversible realization of quaternary decoder, multiplexer, and demultiplexer circuits. Eng. Lett., 15: 203-207. $\mathrm{http}: / /$ search.ebscohost.com/login.aspx?direct=true $\& \mathrm{db}=\mathrm{a} 9 \mathrm{~h} \& \mathrm{AN}=27952764 \&$ site $=$ ehost-live

5. Rakes, C.D., 1998. CMOS ICsdecoders and multiplexers. Popular Electr., 15: 66-49. http://search.ebscohost.com/login.aspx?direct=true $\& \mathrm{db}=\mathrm{a} 9 \mathrm{~h} \& \mathrm{AN}=232579 \&$ site $=$ ehost-live 